\author{
Jean-Michel Jeannin
}

Einleitung

Bei Centella asiatica (Hydrocotyle asiatica, Gotu Kola, Brahmi, Indischer Wassernabel) (Abb. 1) handelt es sich um eine krautige Blütenpflanze aus der Familie der Doldengewächse. Die Blätter sind glatt, nierenförmig, gelappt oder gekerbt und haben einen Durchmesser von 1,3-6,3 cm. Die Blütenstände umfassen 2-3 Dolden, die ihrerseits 1-5 einzelne weisse oder rötliche Blüten aufweisen. Die länglichen, kleinen Früchte zeigen eine Primär- und eine Sekundärrippung. Die Pflanze gedeiht in Wassernähe und an sumpfigen Stellen [1]. Sie stammt aus Madagaskar, dem tropischen Asien, Mittel- und östlichem Südamerika und ist auch in Australien und Südafrika heimisch $[1,2]$.

\section{Verwendung}

Als Droge werden die gesamten oberirdischen Bestandteile oder auch nur die Blätter verwendet. Extrakte werden nach dem Asiaticosid-Gehalt standardisiert. Die lokale Applikation zur Behandlung von Wunden, Narben und Verbrennungen ist durch klinische Studien gut belegt. Ebenso belegt ist die perorale Anwendung zur Behandlung von Magendarmulzera. In Europa ist die perorale Verabreichung zur Behandlung der venösen Insuffizienz gebräuchlich (z.B. Madecassol ist unter anderem in Frankreich zugelassen).

Die traditionelle Verwendung umfasst unter anderem die Behandlung von Hirnleistungsstörungen im Alter sowie von Lepra, Syphilis, Tuberkulose, Varizellen, Verstopfung, Durch-

\title{
Exotische Heilpflanzen (7)
}

\author{
Centella asiatica (L.) Urban (Apiaceae)
}

fall, Hepatitis, Epilepsie usw. Der Indische Wassernabel wird in China auch als Antidot bei Arsenvergiftung und Schlangenbissen verwendet [2]. Die Blätter werden in Bangladesch, Thailand und Sri Lanka als VitaminC- und Carotin-reiches sowie proteinhaltiges Blattgemüse verkauft [2]. In den USA wird C. asiatica als Nahrungsergänzungsmittel zur Verbesserung der Gedächtnisleistung propagiert [2]. Extrakte der Blätter findet man auch in Kosmetika (Cremes, Shampoos, Haarfestiger) [2].

\section{Phytochemie}

Die Inhaltsstoffe variieren je nach Chemotyp. Die Blätter enthalten $1,1-8,0 \%$ triterpenoide Saponine, darunter die Triterpenglycosidester Asiaticosid und Madecassiosid sowie Oxiasiaticosid (Chemotyp Madagaskar), Centellosid (Chemotyp Sri Lanka), Brahmosid, Brahminosid, Thankunosid, Isothankunosid (Chemotyp Indien). Verschiedene Chemotypen enthalten Asiat-, Madekassia-, Centella-, Indocentoat-, Brahmat-, Thankunat- und Isothankunatsäure sowie das spezifische Oligosaccharid Centellose. Die Gesamtpflanze enthält ferner 13,8 mg/100 g Trockengewicht Ascorbinsäure [2].

\section{Aktuelle Forschung}

Die aktuelle Forschung befasst sich mit der antimikrobiellen, zytotoxischen und antioxidativen Wirkung [3], der Wirkung bei venöser Insuffizienz sowie der Verwendung zur Verbesserung der Hirnleistung [2, 4, 5].

\section{Literatur}

1 Herba Centellae; in WHO Monographs on Selected Medicinal Plants, Vol. 1. Geneva, World Health Organization, 1999.

2 Gotu Kola; in Leung AY, Forster S: Encyclopedia of Common Natural Ingredients, ed 2. New York, John Wiley \& Sons, 1996.

3 Ullah MO, et al: Antimicrobial, cytotoxic and antioxidant activity of Centella asiatica. Eur J Sci Res 2009;30:260-264.

4 Mato L, et al: Centella asiatica improves physical performance and health-related quality of life in healthy elderly volunteer. Evid Based Complement Alternat Med 2009; DOI: $10.1093 /$ ecam/nep177.

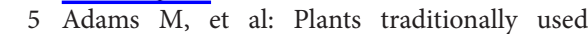
in age-related brain disorders - a survey of ethnobotanical literature. J Ethnopharmacol 2007;113:363-381.
Abb. 1. Centella asiatica. Viktoriahaus des Botanischen Gartens der Universität Basel, 2010 .

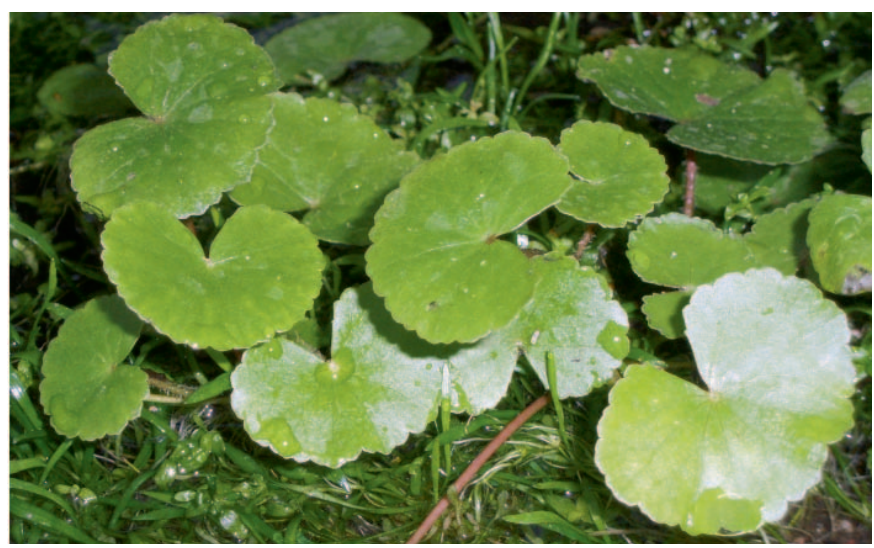

\section{KARGER}

Fax +497614520714 Information@Karger.d www.karger.com
(C) 2011 S. Karger GmbH, Freiburg
Dipl. med. biol. Jean-Michel Jeannin Holeestrasse 43, 4054 Basel, Schweiz Tel. +41 61-4215991, Fax - 4230313 jmjeannin@dataworks.ch 